\title{
Impact of Pulse Shaping Design on OOB Emission and Error Probability of GFDM
}

\author{
Surbhi Kalsotra*, Atul Kumar ${ }^{\dagger}$, Hem Dutt Joshi*, Ashutosh Kumar Singh*, Kapal Dev ${ }^{\ddagger}$ and Maurizio Magarini ${ }^{\ddagger}$ \\ * Department of Communication Engineering, Thapar Institute of Engineering and Technology Patiala, India \\ Email: \{skalsotra_me16, hemdutt.joshi, aksingh\}@thapar.edu. \\ $\dagger$ Vodafone Chair Mobile Communications Systems, Technische Universität Dresden, Germany. \\ Email: atul.kumar@tu-dresden.de. \\ $\ddagger$ Department of Electronic, Information and Bioenginering, Politecnico di Milano, Milano, Italy \\ Email: \{kapal.dev, maurizio.magarini\}@polimi.it.
}

\begin{abstract}
Generalized frequency-division multiplexing is one of the multi-carrier modulation schemes currently under study for beyond fifth generation (B5G) cellular systems. The main characteristics of generalized frequency-division multiplexing (GFDM) are the flexible time-frequency structure of data blocks, making it customizable according to various service requirements in B5G scenarios, and the low out of band (OOB) emission, which can be achieved by means of a flexible time-domain pulse shaping of individual sub-carriers. With this motivation, a new pulse shaping filter is proposed and its benefits on the OOB emission and on the symbol error probability (SEP) are demonstrated. The eye diagram, used as a performance evaluation parameter at the transmitter, shows that the proposed pulse has better vertical sharpness than root-raised cosine (RRC) filter and, therefore, it gives better $O O B$ emission. Along with vertical sharpness, it has better horizontal sharpness, which means that the proposed pulse has better tolerance to inter-symbol interference (ISI). The SEP performance is evaluated by means of Monta Carlo simulations in case of 16-QAM transmission over an AWGN channel. Analytical expressions of SEP are also presented.

Index Terms-Symbol error Probability (SEP), Root-Raised Cosine (RRC), Generalized Frequency Division Multiplexing (GFDM), Out-of-Band (OOB) emissions.
\end{abstract}

\section{INTRODUCTION}

The aim of beyond fifth generation (B5G) wireless communication networks is to increase the quality and quantity of wireless services while keeping high the link reliability and low the latency. This is useful for applications like smart transportation, remote health, remote education, smart grid, virtual, and augmented reality [1]. The main physical layer (PHY) requirements for these applications are: massive concurrent access, high energy efficiency, and low latency with ultra-high reliability [2].

The look for new waveforms that are able to support variable and customizable pulse shaping filters is one of the research priorities in order to achieve a better tradeoff between timedomain and frequency-domain localization. To satisfy such PHY requirements, generalized frequency division multiplexing (GFDM) has been proposed as one of the possible multiplexing techniques for B5G communication. Its main characteristics are low out-of-band (OOB) emission and less cyclic prefix (CP) overhead as compared to orthogonal frequency-division multiplexing (OFDM). It is also able to support variable and customizable pulse shaping filters, which can be used as one of the research priorities in order to achieve a better trade-off between time-domain and frequency-domain localization [3].

Basically, GFDM consists of blocks of data having independently modulated waveforms, where every block consists of various sub-carriers and every sub-carrier has various subsymbols [3]. A filtering is done at sub-carrier level by using circularly shifted versions of a prototype filter, which is shifted in both domains: time as well as frequency [4]. The choice of the pulse shaping has a strong impact on OOB emission and symbol error probability (SEP) performance of the GFDM system. As a result of the filtering the orthogonality among the sub-carriers is lost.

Several works are available in the literature that focus on the impact of the pulse shaping filter on the GFDM performance [5]-[9]. In [5], the effect of pulse shaping on OOB emission and bit-error rate (BER) is studied for the additive white Gaussian noise (AWGN) channel. It is shown that GFDM has lower OOB emission as compared to OFDM and its BER equals that of OFDM when Dirichlet prototype filter is used. Moreover, in [6]-[8] improved Nyquist pulse shaping filters are used in combination with zero forcing ( $\mathrm{ZF})$ receiver, which give better SEP performance than RRC. By using Meyer auxiliary function along with pulse shaping filter, there is further improvement in BER and OOB emission. Most recent work in [9] proposes a new ramp-based filter that has OOB emission lower than RRC but higher than Xia pulses but provides better SEP performance. In this paper we propose the design of a new pulse shaping filter that results from the linear combination of the two pulse shapes and analyze the OOB emission and SEP performance with $\mathrm{ZF}$ and $\mathrm{MF}$ and MMSE receiver in the case of the transmission over the AWGN channel.

The paper is organized as follows. Section II presents the system model. The role of the pulse shaping filter is illustrated in Sec. III, while Sec. V presents the evaluation of the signalto-interference-plus-noise ratio (SINR). Simulation results are shown in Sec. VI and, finally, Sec. VII draws the conclusion. 


\section{System Model}

With reference to GFDM symbol [4], a block of transmitted bits is applied to the input of a mapper which produces at its output the vector $\mathbf{D}$ which can be decompose into $K$ subcarriers having $M$ sub-symbols.

$$
\mathbf{D}=\left[\mathbf{d}_{0}^{T}, \mathbf{d}_{1}^{T}, \cdots, \mathbf{d}_{K-1}^{T}\right]^{T},
$$

where the time-duration of each symbol vector $\mathbf{d}_{k}$ is $M T_{s}$, with $T_{s}$ is the symbol interval on each sub-carrier. The subcarrier spacing equal to $1 /\left(M T_{s}\right)$. The $M$ sub-symbols of the $k$ th sub-carrier are represented by the vector

$$
\mathbf{d}_{k}=\left[d_{k, 0}, d_{k, 1}, \cdots, d_{k, M-1}\right]^{T},
$$

where $d_{k, m}$ is the data transmitted on the $k$ th sub-carrier and $m$ th sub-symbol. Therefore, the total number of symbols present in a GFDM block is $N=K M$. Thereafter, pulse shaping is performed on each data symbol separately and the $n$th sample of the transmitted GFDM signal after pulse shaping is

$$
x(n)=\sum_{k=0}^{K-1} \sum_{m=0}^{M-1} p_{k, m}[n] d_{k, m},
$$

with

$p_{k, m}[n]=p[(n-m K) \bmod N] e^{\frac{j 2 \pi k n}{K}}, n=0,1, \ldots, N-1$,

where $p[n]$ is the pulse shaping filter of of length $N$ and $\bmod N$ is the modulo $N$ operation.

After the pulse shaping filter, the sequence (3) is transmitted over an ideal AWGN channel and the received signal is given by

$$
y(n)=x(n)+w(n), n=0,1, \ldots, N-1,
$$

where $w(n)$ is the white Gaussian noise with variance $\sigma^{2}$.

In order to have a more compact notation, a matrix representation is adopted. The vector $N \times 1$ vector of received samples is

$$
\mathbf{y}=\mathbf{B D}+\mathbf{w}
$$

where $\mathbf{y}=[y[0], y[1], \ldots, y[M K-1]]^{T}, \mathbf{B}=\left[\mathbf{p}_{0,0} \cdots \mathbf{p}_{K-1,0}\right.$, $\left.\mathbf{p}_{0,1} \cdots \mathbf{p}_{K-1,1}, \cdots, \mathbf{p}_{0, M-1} \cdots \mathbf{p}_{K-1, M-1}\right]$ in which $\mathbf{p}_{k, m}=\left[p_{k, m}[n]\right]^{T}$ and $\mathbf{w}$ is the noise vector in which each entry is an i.i.d. zero mean complex Gaussian random variable with noise power $\sigma^{2}$

By following [7], different linear receiver schemes are considered to demodulate the received signal. The data matrix at after linear processing is written as

$$
\hat{\mathbf{D}}=\boldsymbol{\Lambda}_{R x} \mathbf{y},
$$

where $\boldsymbol{\Lambda}_{R x}$ is demodulation matrix. Depending on the receiver architecture, its expression is going to change. The standard linear receivers used for demodulation are: Zero Forcing (ZF), Matched Filter (MF), and Minimum Mean-Squared Error (MMSE) [10], [11]. The details of the each receiver are describe below:

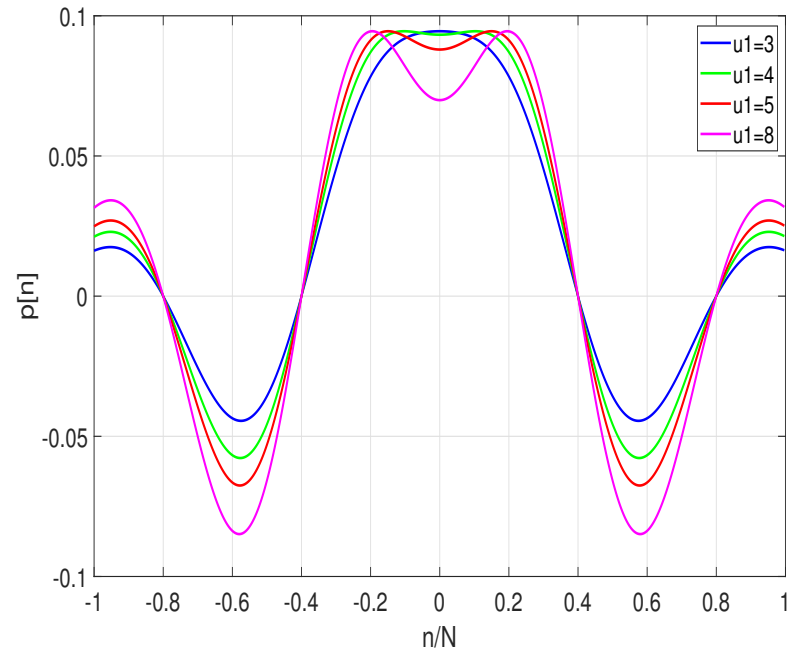

Figure 1. Impulse response of proposed pulse shaping filter for $u>2$.

- ZF Receiver removes the ISI and ICI at the receiver, but with the possible noise enhancement which effects the SINR performance. As the noise enhancement depends upon pulse shaping, a proper design of the prototype filter makes possible to improve the SEP performance. In the case of $\mathrm{ZF}$ receiver the expression of the demodulation matrix is

$$
\mathbf{\Lambda}_{Z F}=\mathbf{B}^{-1} \text {. }
$$

Finally, the demodulated signal is written as

$$
\hat{\mathbf{D}}=\boldsymbol{\Lambda}_{Z F} y=\mathbf{B}^{-1}(\mathbf{B D}+\mathbf{w}) \text {. }
$$

- MF Receiver maximizes the signal-to-noise ratio (SNR) but introduces ISI and inter-carrier interference (ICI) when the applied transmit pulse is non-orthogonal. In this receiver, the linear receiving matrix is given by

$$
\boldsymbol{\Lambda}_{M F}=\mathbf{B}^{\mathbf{H}}
$$

and the demodulated signal is

$$
\hat{\mathbf{D}}=\boldsymbol{\Lambda}_{M F} y=\mathbf{B}^{H}(\mathbf{B D}+\mathbf{w}) .
$$

- MMSE Receiver gives the best trade-off between noise enhancement and self-interference. In this receiver, the demodulation matrix is given by

$$
\boldsymbol{\Lambda}_{M M S E}=\left(\frac{\sigma^{2}}{E_{s}} I+\mathbf{B}^{H} \mathbf{B}\right)^{-1} \mathbf{B}^{H},
$$

where $E_{s}$ is the signal energy and $I$ is the identity matrix. Finally, demodulated signal after the MMSE receiver is given by

$$
\hat{\mathbf{D}}=\boldsymbol{\Lambda}_{M M S E} y=\left(\frac{\sigma^{2}}{E_{s}} I+\mathbf{B}^{H} \mathbf{B}\right)^{-1} \mathbf{B}^{H}(\mathbf{B D}+\mathbf{w}) \text {. }
$$

The demodulated output $\hat{\mathbf{D}}$ obtained with the different linear receiver schemes is given to the QAM de-mapper that takes a decision about transmitted bits. 

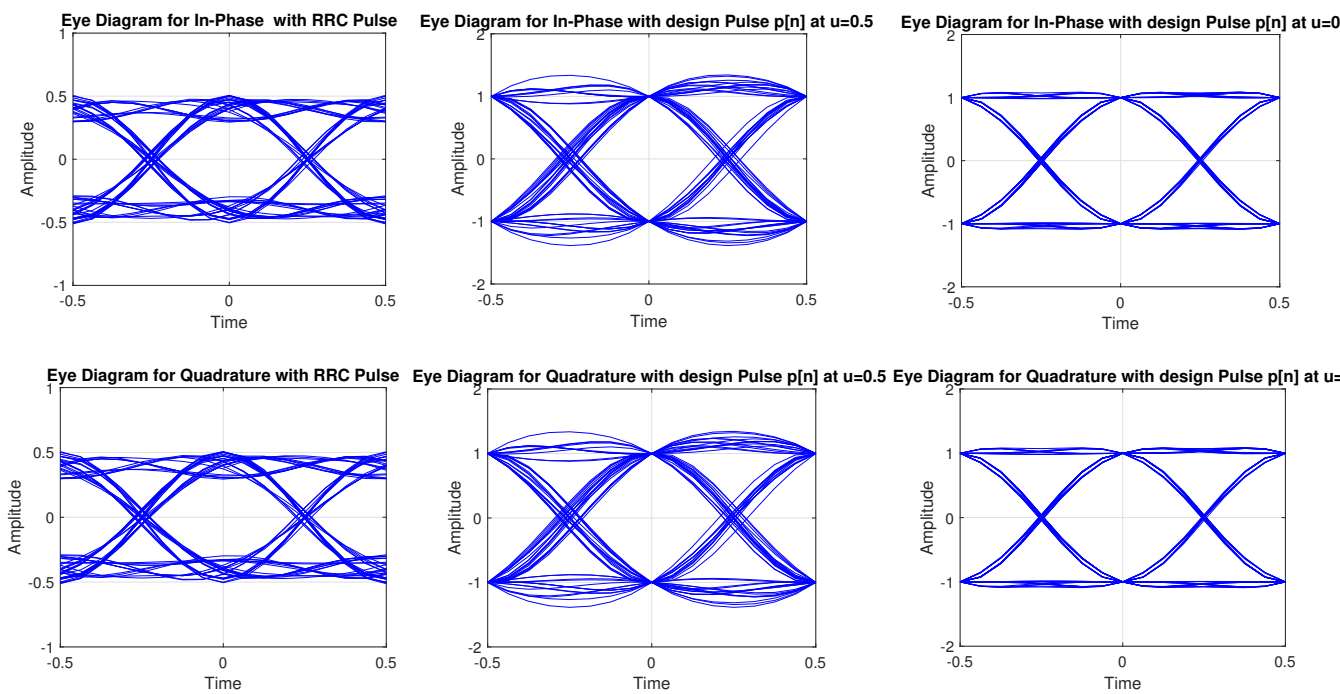

Figure 2. Eye diagram comparison of the design pulse with RRC pulse with different values of the design parameters.

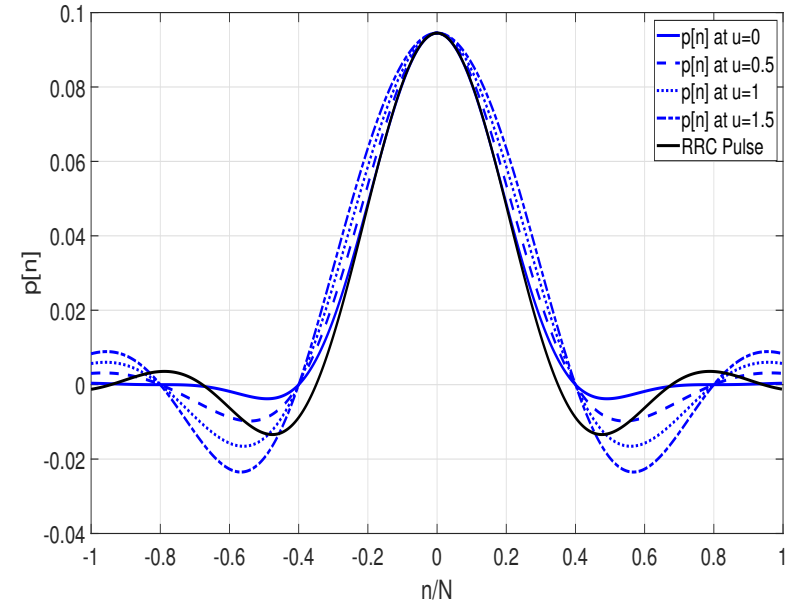

Figure 3. Impulse response of proposed filter and RRC filter for alpha equal to 0.5 .

\section{Pulse Shaping Filter}

The choice of the pulse shaping filter $p[n]$ strongly influences the spectral properties of the GFDM signal. Here, we consider the classical approach of choosing the discrete time impulse response by sampling a continuous-time impulse response $p(t)$. As impulse response $p(t)$ we consider a linear combination of two pulses

$$
p(t)=(1-u) p_{1}(t)+u p_{2}(t),
$$

where $u$ is the constant that corresponds to linear combination of two pulses having range $[0,2]$. The choice of $u$ provides an extra degree of freedom to pulse shaping. As the value of $u$ starts increasing beyond 2, the envelope of pulse shape starts changing and the pulse shape will no longer behave as nyquist pulse shape as shown in Figure 1. For $u=3$ the main lobe of the pulse starts becoming broad and for the value of $u=4,5$ and 8 as shown in Figure 1, the pulse central value will no longer remain maximum. The pulses $p_{1}(t)$ and $p_{2}(t)$ used in (14) are given as [12]

$$
p_{1}(t)=\operatorname{sinc}(t / T)(\operatorname{sinc}(\alpha t / T))^{2}
$$

and

$$
p_{2}(t)=\operatorname{sinc}(t / T)(\operatorname{sinc}(\alpha t / 2 T))
$$

The (14) can also be rewritten as

$$
p(t)=\frac{\sin (\pi \tau)}{\pi \tau} \frac{(1-u) \sin ^{2}(\pi \alpha \tau)+2 u \pi \alpha \tau \sin (\pi \alpha \tau / 2)}{(\pi \alpha \tau)^{2}}
$$

where $\tau=t / T$ is the time normalization. The continuoustime impulse response of the new pulse shape is compared with the RRC pulse shape filter in Figure 3. From this figure, it is clear that as value of $u$ increases, the side lobe levels of the proposed pulse shape increases, as also confirmed by the eye diagrams shown in Fig. 2.

\section{Power Spectral Density}

Power Spectral Density (PSD) is defined as the content of power in the signal versus the frequency range. GFDM signal has coefficient of pulse shaping filters as one of its component. So, OOB emissions can be reduced by selecting the appropriate pulse shaping filter [5].

$$
P S D(f)=\lim _{T \rightarrow \infty}\left(\frac{1}{T} E\left(\left|F\left(x_{T}(t)\right)\right|^{2}\right)\right)
$$

where $T$ denotes the time period of the signal and $x_{T}$ is the truncated GFDM signal at interval $(-T / 2, T / 2)$. 


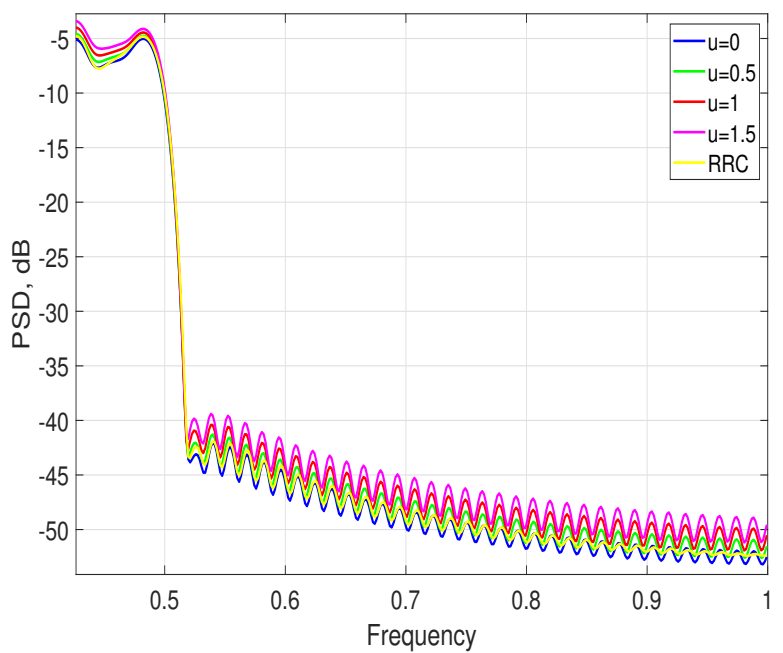

Figure 4. PSD for transmitted GFDM signal for $\alpha=0.5$.

\section{Signal-TO-INTERFERENCE-PLUS-NOISE RATIO}

In this section, the analysis of the SINR is given. The resulting expression is used to calculate the SEP [13]. The general expression of the linearly demodulated signal is

$$
\hat{\mathbf{D}}=\boldsymbol{\Lambda}_{R x}(\mathbf{B d}+\mathbf{w})=\boldsymbol{\Lambda}_{R x} \mathbf{B D}+\boldsymbol{\Lambda}_{R x} \mathbf{w},
$$

where $\boldsymbol{\Lambda}_{R x}$ is the GFDM demodulation matrix. Depending on the type of the pulse shaping filter and of the receiver architecture, the expression in (19) can be decomposed as

$$
\hat{\mathbf{D}}=\text { Desired signal }+ \text { ISI }+ \text { ICI }+\mathbf{w} .
$$

The computation of the average power of each term plays an important role in the SINR modeling. The average power of the desired signal is calculated by squaring the average of all the diagonal elements of the matrix $\boldsymbol{\Lambda}_{R x} \mathbf{B}$ which is given as

$$
P_{s}=E_{s}\left|\frac{1}{N} \sum_{k=0}^{N-1}\left[\boldsymbol{\Lambda}_{R x} \mathbf{B}\right]_{(k, k)}\right|^{2},
$$

The average ISI power is calculated by subtracting the average power of desired signal from the average of the total signal power due to the diagonal elements of the matrix $\boldsymbol{\Lambda}_{R x} \mathbf{B}$ which is given by the equation

$$
I_{\text {ISI }}=\frac{E_{s}}{N} \sum_{k=0}^{N-1}\left|\left[\boldsymbol{\Lambda}_{R x} \mathbf{B}\right]_{(k, k)}\right|^{2}-P_{s},
$$

The ICI occurs due to the non-orthogonality in the system and its average power is calculated by averaging the square of non-diagonal elements of the matrix $\boldsymbol{\Lambda}_{R x} \mathbf{B}$ and it is given as

$$
I_{\mathrm{ICI}}=\frac{E_{s}}{N} \sum_{k=0}^{N-1} \sum_{m=0, m \neq k}^{N-1}\left|\left[\boldsymbol{\Lambda}_{R x} \mathbf{B}\right]_{(k, m)}\right|^{2},
$$

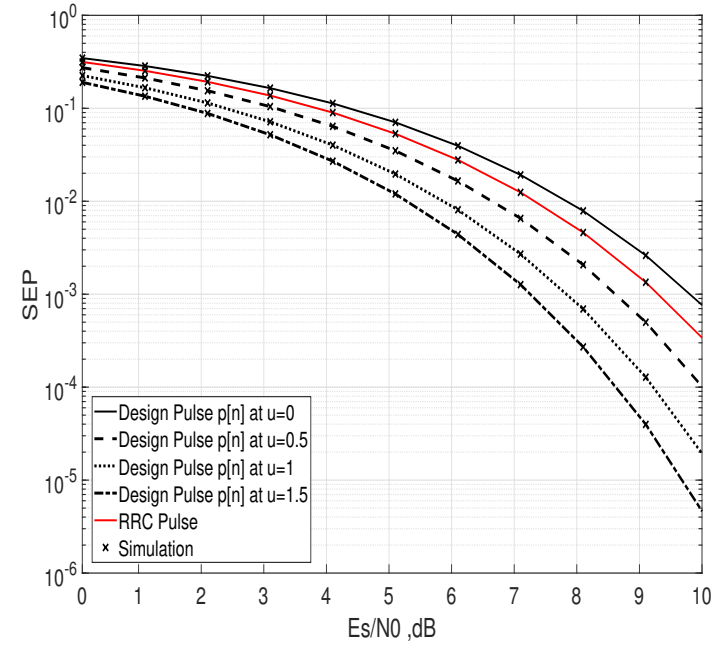

Figure 5. SEP vs. $E s / N 0$ of GFDM signal for $\alpha=0.5$ using ZF receiver in case of 16-QAM transmission over AWGN.

The average noise power is calculated by averaging the square of the elements of the matrix $\Lambda_{R x}$ and is given by the equation

$$
\sigma_{n}^{2}=\frac{\sigma^{2}}{M}\left[\frac{1}{N} \sum_{k=0}^{N-1} \sum_{m=0}^{N-1}\left|\left[\boldsymbol{\Lambda}_{R x}\right]_{(k, m)}\right|^{2}\right],
$$

where $\sigma^{2}$ is the additive noise power and $\sigma^{2}=N_{0} / 2$. Therefore, the expression of the SINR is written as

$$
\mathrm{SINR}=\frac{P_{s}}{I_{I S I}+I_{I C I}+\sigma_{n}^{2}}
$$

By assuming that the overall interference term can be approximated as a Gaussian random variable, its power can be added to that of the noise one. The signal-to-noise ratio (SNR) is given by $E_{s} / N_{0}$ where $E_{s}$ is the mean power and $N_{0}$ is the variance for the AWGN. Therefore, the symbol error probability for QAM modulation in the case of the transmission over AWGN channel is given by

$$
\mathrm{SEP}=2\left(\frac{k-1}{k}\right) \operatorname{erf} f_{c}(\sqrt{\gamma})-\left(\frac{k-1}{k}\right) \operatorname{erf} f_{c}^{2}(\sqrt{\gamma})
$$

where $\gamma$

$$
\gamma=\frac{3 R_{T}}{2\left(2^{\mu}-1\right)} S N R
$$

with

$$
R_{T}=\frac{K M}{K M+N_{c p}+N_{c s}}
$$

and $\mu$ is the number of bits per QAM symbol, $k=\sqrt{2^{\mu}}, N_{c p}$ and $N_{c s}$ are the length of cyclic prefix and cyclic suffix.

\section{Simulation Results}

In this section, results for GFDM obtained by using the design pulse shaping filter in Sec. III is presented and compared with baseline pulse shaping filter used in GFDM known as root-raised-cosine filter. Table I reports the parameters used in 


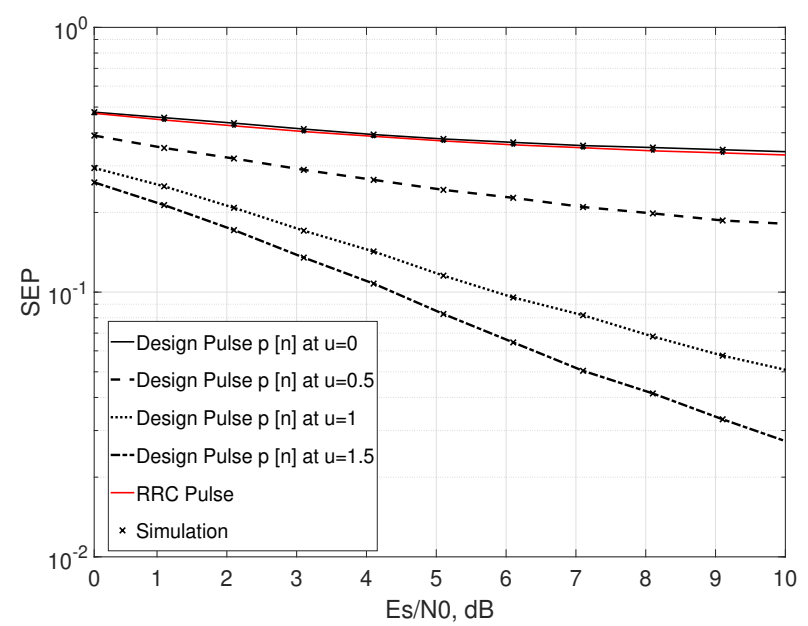

Figure 6. SEP vs. Es $/ N 0$ of GFDM signal for $\alpha=0.5$ using MF receiver in case of 16-QAM transmission over AWGN..

the simulations. The analytical results of the SEP verse Es/No performance with roll-off $(\alpha)=0.5$ is verify by the Monte Carlo simulation (marked as $\times$ ).

Table I

Simulation PARAMETERS

\begin{tabular}{|l|c|}
\hline Sampling frequency & $1.92 \mathrm{MHz}$ \\
\hline Sampling time & $0.52 \mu \mathrm{s}$ \\
\hline Symbol duration & $66.6 \mu \mathrm{s}$ \\
\hline Samples per symbol & 128 \\
\hline Sub-symbols(M) & 5 \\
\hline Sub-carriers $(\mathrm{K})$ & 128 \\
\hline Mapping & $16-\mathrm{QAM}$ \\
\hline Roll off factor $(\alpha)$ & 0.5 \\
\hline Cyclic Prefix $(\mathrm{CP})$ length & 32 \\
\hline Channel & AWGN \\
\hline
\end{tabular}

Figure 4 shows the simulation result of PSD of GFDM signal for $\alpha=0.5$. The side lobe level of the proposed pulse shape decreases with increase in $u$. So, the OOB emission is lower for $u=0$ when compared with other values of $u$. It can also be seen from the Figure 2 that RRC pulse shaping has better vertical sharpness than new pulse shape at $u=0.5$. But at $u=0$ the new pulse shape has better vertical sharpness compared to RRC. Therefore, the new pulse shape has better OOB emission than RRC at $u=0$.

Figure 5 shows the simulation results of SEP versus $E_{s} / N_{0}$ at $\alpha$ equal to 0.5 for ZF receiver. In case of GFDM transmission through AWGN channel, noise enhancement decreases as value of $u$ increases. So, the proposed pulse shape has lesser value of noise enhancement factor at $u=1.5$ and hence the better SEP results at $u=1.5$. From the Table II at $E_{s} / N_{0}=8 \mathrm{~dB}$, the GFDM system has $S E P=0.006594$ at $u=0$ which decreases to 0.0002656 at $u=1.5$. The SEP results of the new pulse shape are also compared with the RRC pulse shaping which shows that the new pulse shape has better SEP results than RRC pulse shaping except at $u=0$.

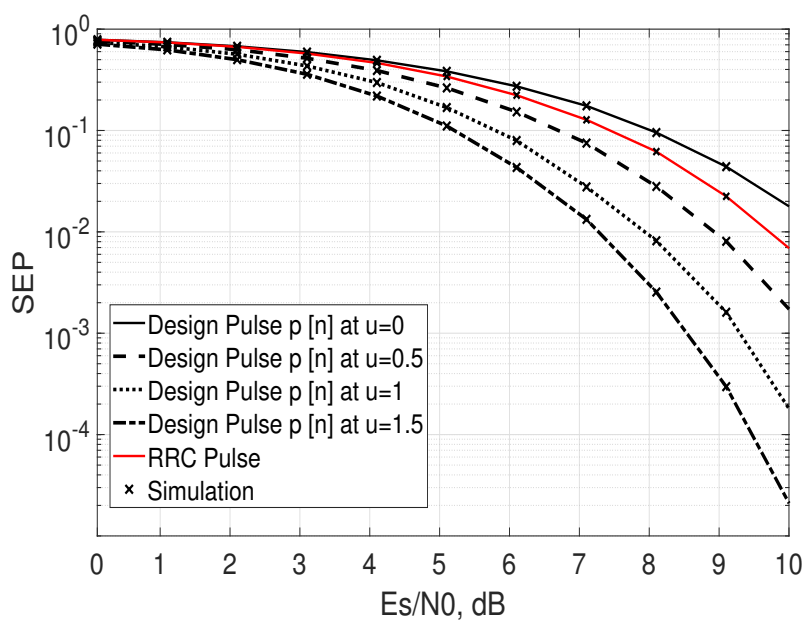

Figure 7. SEP vs. Es/NO of GFDM signal for $\alpha=0.5$ using MMSE receiver in case of 16-QAM transmission over AWGN.

Figure 6 shows the simulation results of SEP versus $E_{s} / N_{0}$ for $\alpha$ equal to 0.5 for MF receiver. While transmitting the GFDM signal through wireless channel, ICI and noise power decreases whereas ISI power increases as value of $u$ increases. The pulse shape side lobe levels has direct relation with ISI in the system. It is clear from the Figure 3 that at $u=0$ the pulse shape has lesser side lobe levels which means having lower ISI value. The ICI occurs due to non-orthogonality in the system which increases with the value of $u$. The effect of ISI is very minimal in comparison to ICI and noise power. It is clear from the Figure 6 that GFDM has best SEP results at $u=1.5$. At $E_{s} / N_{0}=8 \mathrm{~dB}$, the GFDM system has $S E P=0.3516$ at $u=0$ which decreases to 0.04142 at $u=1.5$. The SEP results of the new pulse shape are also compared with the RRC pulse shaping which shows that the new pulse shape has better SEP results than RRC pulse shaping except at $u=0$.

Figure 7 shows the simulation results of SEP versus $E_{s} / N_{0}$ for $\alpha$ equal to 0.5 for MMSE receiver. In this, ICI and noise power decreases and ISI power increases with increase in $u$. The pulse shape side lobe levels has direct relation with ISI in the system. But the effect of ISI is very minimal in comparison to ICI and noise power. It is clear from the Figure 7 that GFDM has best SEP results at $u=1.5$. Also, at $E_{s} / N_{0}=$ $8 \mathrm{~dB}$, the GFDM system has $S E P=0.09542$ at $u=0$ which decreases to 0.002547 at $u=1$.5. The SEP results of the new pulse shape are also compared with the RRC pulse shaping which shows that the new pulse shape has better SEP results than RRC pulse shaping except at $u=0$.

Table II shows the SEP of GFDM signal having 16-QAM transmission in AWGN channel while using different receivers for roll-off factor equal to 0.5. The SER of proposed pulse shape is compared with RRC pulse shaping filter at $E_{s} / N_{0}=$ $8 \mathrm{~dB}$. The proposed pulse shape has better SEP than RRC pulse shaping except at $u=0$ for all three types of linear demodulators. Take an example for $u=1.5$, the ZF receiver has $S E P=0.0002656$ and MF receiver has $S E P=0.04142$ 
whereas the MMSE receiver has $S E P=0.002547$ at $E_{s} / N_{0}=8 \mathrm{~dB}$. So, on comparing the value of SEP for these three receivers, $\mathrm{ZF}$ has lesser value of SEP than other two receivers.

Table II

SEP COMPARISON TABLE

\begin{tabular}{|l|c|c|c|}
\hline Pulse Shape & ZF & MF & MMSE \\
\hline RRC & 0.004016 & 0.3417 & 0.06192 \\
\hline$u=0$ & 0.006594 & 0.3516 & 0.09542 \\
\hline$u=0.5$ & 0.001375 & 0.1983 & 0.02803 \\
\hline$u=1$ & 0.0004375 & 0.06797 & 0.008516 \\
\hline$u=1.5$ & 0.0002656 & 0.04142 & 0.002547 \\
\hline
\end{tabular}

\section{CONCLUSION}

A new pulse shape which is a linear combination of two pulses is proposed in this paper and its impact is studied on OOB emission and SEP for 16-QAM transmission in AWGN channel with $\mathrm{ZF}, \mathrm{MF}$ and MMSE receivers. In this proposed pulse shape, there is a constant value $u$ which provides an extra degree of freedom and by changing its value we can get different pulses. As the value of $u$ increases, the side lobe level increases. OOB emission increases with increase in side lobe level, Also, as value of $u$ increases, vertical sharpness of the receiver eye diagram decreases. So, OOB emission increases with an ncrease in value of $u$.

The SEP in GFDM system depends on ISI, ICI and noise power. The ISI and ICI occur due to non-orthogonality in the GFDM system. The pulse shape side lobe levels has direct relation with the ISI in the system which increases due to increase in side lobe level. For MF and MMSE receiver, the ICI and noise power decreases whereas ISI increases with increase in $u$ but the ISI has negligible effect on the SER and for ZF receiver there is only noise factor which decreases with increase in $u$. Therefore, SEP performance improves with increase in $u$. The SEP results of the new pulse shaping filter give better SEP results except at $u=0$ when compare with the RRC pulse shaping filter. The SEP simulation results are also verified using Mote Carlo simulation.

\section{REFERENCES}

[1] J. Kim, J. Lee, J. Kim, and J. Yun, "M2m service platforms: Survey, issues, and enabling technologies," IEEE Communications Surveys \& Tutorials, vol. 16, no. 1, pp. 61-76, 2014.

[2] H. Ji, S. Park, J. Yeo, Y. Kim, J. Lee, and B. Shim, "Ultra-reliable and low-latency communications in 5g downlink: Physical layer aspects," IEEE Wireless Communications, vol. 25, no. 3, pp. 124-130, 2018.

[3] A. Kumar and M. Magarini, "On the modelling of inter-sub-symbol interference in gfdm transmission," IEEE Communications Letters, pp. 1$1,2019$.

[4] N. Michailow, M. Matthé, I. S. Gaspar, A. N. Caldevilla, L. L. Mendes, A. Festag, and G. Fettweis, "Generalized frequency division multiplexing for 5th generation cellular networks," IEEE Transactions on Communications, vol. 62, no. 9, pp. 3045-3061, 2014.

[5] M. Matthé, N. Michailow, I. Gaspar, and G. Fettweis, "Influence of pulse shaping on bit error rate performance and out of band radiation of generalized frequency division multiplexing," in 2014 IEEE International Conference on Communications Workshops (ICC), pp. 43-48, IEEE, 2014.
[6] A. Kumar and M. Magarini, "Improved nyquist pulse shaping filters for generalized frequency division multiplexing," in 2016 8th IEEE LatinAmerican Conference on Communications (LATINCOM), pp. 1-7, IEEE, 2016.

[7] A. Kumar, M. Magarini, and S. Bregni, "Impact of "better than nyquist" pulse shaping in gfdm phy with lte-compatible frame structure," in 2017 IEEE 9th Latin-American Conference on Communications (LATINCOM), pp. 1-6, IEEE, 2017.

[8] A. Kumar, M. Magarini, and S. Bregni, "Improving gfdm symbol error rate performance using "better than nyquist" pulse shaping filters," IEEE Latin America Transactions, vol. 15, no. 7, pp. 1244-1249, 2017.

[9] M. Mishra, S. Aich, H.-C. Kim, and P. M. Pradhan, "A novel rampbased pulse shaping filter for reducing out of band emission in $5 \mathrm{~g}$ gfdm system," in TENCON 2018-2018 IEEE Region 10 Conference, pp. 00960101, IEEE, 2018.

[10] N. Michailow, S. Krone, M. Lentmaier, and G. Fettweis, "Bit error rate performance of generalized frequency division multiplexing," in 2012 IEEE Vehicular Technology Conference (VTC Fall), pp. 1-5, IEEE, 2012.

[11] I. Gaspar, L. Mendes, M. Matthé, N. Michailow, A. Festag, and G. Fettweis, "Lte-compatible $5 \mathrm{~g}$ phy based on generalized frequency division multiplexing," in 2014 11th International Symposium on Wireless Communications Systems (ISWCS), pp. 209-213, IEEE, 2014.

[12] N. C. Beaulieu and M. O. Damen, "Parametric construction of nyquist-i pulses," IEEE Transactions on communications, vol. 52, no. 12, pp. 2134 2142, 2004.

[13] A. Nimr, M. Chafii, and G. Fettweis, "Practical gfdm-based linear receivers," pp. 1-6, 2019. 